\title{
VORWORT ZUR RUSSISCHEN AUFLAGE
}

Das vorliegende Buch, das wir der Beachtung des Lesers empfehlen, trägt die Benennung ,,Kinematik der Kernreaktionen“. Wir verstehen unter Kinematik die Gesamtheit der Beziehungen, die auf den Erhaltungssätzen beruhen, die ihrerseits aus den Symmetrieeigenschaften von Raum und Zeit in der klassischen und in der Quantenform folgen.

Das Buch besteht aus zwei Teilen. In den beiden Einführungskapiteln, die den ersten Teil eröffnen, wird der Leser kurz an einige Grundcharakteristika der Bewegungen mit relativistischen Geschwindigkeiten erinnert. Außerdem werden dort die für die weitere Darlegung wichtigen relativistischen Transformationen entwickelt. Auch werden zwei Koordinatensysteme definiert, die im weiteren breite Anwendung finden: das Laborsystem und das Schwerpunktsystem. Es werden Formeln für den Übergang aus einem System in das andere angegeben.

Die folgenden Kapitel des ersten Teils sind der klassischen Kinematik der Wechselwirkungen (Stöße' und Zerfälle) mit der Bildung von zwei, drei und mehr Teilchen gewidmet. Wenn im Endzustand nur zwei Teilchen vorhanden sind, so existiert entweder ein vollständig bestimmter Zusammenhang zwischen den Winkeln, unter denen sie emittiert werden, oder zwischen dem Emissionswinkel eines Teilchens und seiner Energie. Die Beziehungen, welche diesen Zusammenhang charakterisieren, werden sowohl in analytischer wie auch in graphischer Form gegeben. Gesondert besprochen werden spezielle Fälle von nichtrelativistischen Wechselwirkungen und von Umwandlungen, an denen Photonen teilnehmen. Werden im Endzustand drei oder mehr Teilchen gebildet, so ist der Zusammenhang zwischen deren Emissionsrichtungen und der Energie nicht eindeutig. Man muß sich in diesen Fällen auf verschiedene Extremalbeziehungen beschränken. Die Betrachtung der Winkel- und Energieverteilungen bei Vielfachprozessen wird auf der Grundlage der Fermrschen statistischen Theorie geführt. Die Aufnahme dieses Abschnittes in ein Búch über Kinematik kann dadurch gerechtfertigt werden, daß in Übereinstimmung mit der ursprünglichen FeRmischen Konzeption die WinkelEnergieverteilung von Vielfachprozessen auf die Erhaltungssätze für Energie und Impuls zurückgeführt werden können.

Der zweite Teil des Buches ist der quantenmechanischen Betrachtung der Kinematik von Kernreaktionen gewidmet.

In diesem Teil wird einer der fundamentalen Begriffe analysiert, dessen Bedeutung bei der Interpretation von Kernwechselwirkungen ständig wächst. Es ist dies der Begriff der Streumatrix oder S.Matrix, der von W. HeIsenberg eingeführt wurde. Es werden die Haupteigenschaften dieser Matrix untersucht. Auf derGrundlage der $S$-Matrix-Theorie und der DiRacschen Transformationstheorie werden die mit den Erhaltungssätzen zusammenhängenden Eigenschaften der Wirkungsquerschnitte von Kernreaktionen untersucht. Die im Buch benutzte Diracsche Transformationstheorie ermöglicht eine leichtverständliche Einführung - ohne Benutzung der Gruppentheorie - der verschiedenen Vektor-Additions-Koeffi- 
zienten, die in der Theorie der Kernreaktionen Anwendung finden (ClebschGordan-Koeffizienten, RACAH-Koeffizienten, Z-Koeffizienten, $X$-Koeffizienten).

Verhältnismäßig viel Raum haben wir dem Problem der Zeitinversion in der Quantenmechanik ( $\$ 21)$ gewidmet. In der Mehrzahl der Arbeiten zur allgemeinen $S$-Matrix-Theorie, einschließlich der klassischen Arbeiten, sind nämlich eine Reihe von Fehlern enthalten, die damit zusammenhängen, daß diese Frage der Zeitinversion nicht akkurat betrachtet wurde. Diese Fehler sind schon so fest in die Theorie eingegangen, daß man gegenwärtig diesen Problemkreis genau kennen muß, um bei der Anwendung von Formeln aus der Literatur Mißverständnisse zu vermeiden. Der wichtige Spezialfall von Kernreaktionen, an denen Photonen teilnehmen, wird gesondert betrachtet.

Der zweite Teil des Buches beschränkt sich auf die Behandlung der eigentlichen Kernreaktionen. Es wurden keine Probleme aufgenommen, die mit dem Zerfall von Teilchen zusammenhängen (z. B. Fragen der Korrelationen beim Zerfall). Ebensowenig werden Reaktionen mit polarisierten Teilchen untersucht. Es wurden lediglich die Fragen der Entstehung polarisierter Teilchen bei Kernreaktionen und die damit verbundenen Hauptgesetzmäßigkeiten behandelt. Um diese fortgelassenen Fragen behandeln zu können, wäre es notwendig gewesen, den Aufwand an mathematischen Hilfsmitteln zu vergrößern. Das Verständnis des behandelten Stoffes wäre damit erschwert worden. Auch hätte dies zu einer beträchtlichen Erweiterung des Buches geführt. Schließlich ist das Buch hauptsächlich für Experimentatoren bestimmt.

Obwohl wir es durchaus als unser Ziel betrachteten, ein Nachschlagewerk über die Kinematik der Kernreaktionen zu schaffen, so haben wir es doch als zweckmäBig empfunden, in das Buch eine Reihe von Tabellen, Diagrammen, Zahlenangaben und Beispielen aufzunehmen. So sind im Anhang I Diagramme gegeben, die den Zusammenhang zwischen den Winkeln und den Energien für einige genauer untersuchte Wechselwirkungen darstellen, an denen leichte Kerne oder Elementarteilchen beteiligt sind. Im Anhang II sind Tabellen der Clebsch-Gordan- und RACAH-Koeffizienten enthalten, daneben auch Zahlentabellen der $Z-, Z_{\gamma^{-}}$und $X$-Koeffizienten.

Der erste Teil des Buches wurde von W. I. GoldanskiJ und I. L. Rosentax geschrieben, der zweite Teil von A. M. BaLdin.

Es ist uns eine selbstverständliche Pflicht, die umfangreiche Arbeit von W. A. Petrunkin und A. I. Lebedev hervorzuheben, von denen der Anhang II zusammengestellt wurde. Sie haben die Tabellen für die $W$-, $Z$-, $Z_{\gamma^{-}}$und $X$-Koeffizienten überprüft und überarbeitet.

Dieses Buch stellt einen ersten Versuch dar, die Kenntnisse von der Kinematik der Kernreaktionen zu verallgemeinern und zu systematisieren. Wir wissen selbst sehr gut, daß es noch viele Mängel und Unzulänglichkeiten enthält. Wir möchten schon jetzt allen Lesern danken, welche die Arbeit auf sich nehmen, sich genauer mit dem Buch zu beschäftigen und uns ihre Hinweise mitteilen.

Zum Abschluß möchten wir W. B. BerestezkiJ und G. I. KopYlow unsere Dankbarkeit für ihre wertvollen Ratschläge ausdrücken.

Aleksandr Michajloviǒ Baldin, Witalis Iosifoviǒ GoldanskiJ, Iosif Leonidovič Rosental. 